Revista de la Facultad de Ingeniería Industrial

19(1): 33-37 (2016) UNMSM

ISSN: 1560-9146 (Impreso) / ISSN: 1810-9993 (Electrónico)

\title{
Aplicación de las 5S para mejorar la percepción de cultura de calidad en microempresas de confecciones textiles en el Cono Norte de Lima
}

\begin{abstract}
RESUMEN
La investigación, realizada en un micro taller de confecciones textiles, permitió el acercamiento del quehacer universitario con la realidad de las microempresas del cono norte de Lima. Primero se efectuó el diagnóstico de la microempresa, tanto en el aspecto del proceso productivo como en el comercial. Se encontró un taller cuyas operaciones son realizadas en un espacio relativamente reducido, sin las fichas técnicas correspondientes. La aplicación de las $5 \mathrm{~S}$ contó con la participación activa del dueño y personal de la micro empresa, la misma que tuvo mayor tiempo de ejecución en las primeras tres S (seleccionar, seiri; organizar, seiton; y, limpiar, seiso). El hallazgo central de la investigación es la variación positiva y significativa de la percepción de calidad de parte del personal de la micro empresa, dando cuenta de la importancia de la aplicación de esta herramienta.
\end{abstract}

Palabras clave: $5 \mathrm{~S}$, calidad, microempresas textiles

5S IMPLEMENTATION OF PERCEPTION TO IMPROVE QUALITY CULTURE MICROENTERPRISES APPAREL TEXTILES in Northern CONE Of LiMA

\section{ABSTRACT}

The research, conducted on a microtextile manufacturing workshop allowed the approach of university life with the reality of micro enterprises in the Northern Cone of Lima. First diagnosis microenterprise is made, both in terms of production process and commercial. A workshop was found whose operations are conducted in a relatively small space, without corresponding data sheets. The implementation of $5 \mathrm{~S}$ had the active participation of the owner and staff of the micro enterprise, the same that had the most runtime in the first three $S$ (select, seiri, organize, seiton; and clean, seiso). The central finding of the research is positive and significant change in the perceived quality of the staff of the micro enterprise, realizing the importance of the implementation of this tool.

Keywords: $5 S$, quality, micro textiles

\section{INTRODUCCIÓN}

El sector textil y confecciones en el Perú es considerado como uno de los motores de desarrollo y uno de los mayores generadores de empleo. Estadísticas del Ministerio de la Producción muestran que este sector es el más inclusivo, pues aporta el $27 \%$ de la PEA manufacturera, cifra que equivale al $10 \%$ del PBI industrial y el $1.5 \%$ del PBI nacional. (Boletín SNI, 2013).

En relación al programa "Compras MyPerú", el gobierno viene comprando 5.3 millones de prendas al sector privado, lo que ha representado unos S/. 320 millones, siendo unas 3193 MYPE las que se han beneficiado directamente con este programa, constituyendo una alternativa de negocio para las mimypes (micro, medianas y pequeñas empresas).

Respecto al tejido empresarial, las estadísticas son elocuentes: la microempresa representa el $94,2 \%$ (1 270 009) del empresariado nacional, la pequeña empresa es el 5,1\% (68 243 empresas) y la mediana empresa el 0,2\% (2 451 empresas); asimismo, las empresas con ventas menores o iguales a 13 uit representan el $70,3 \%$ del tejido empresarial formal, y en el extremo superior las empresas con ventas mayores a 2300 UIT representan tan solo el $0,6 \%$. De acuerdo al número de trabajadores empleados, el $96,7 \%$ de las mipymes emplea hasta a cinco trabajadores.

Las mipymes formales por tipo de organización, en el 2012, fueron: $80,6 \%$ empresa individual (incluye persona natural y empresa individual de responsabilidad limitada) y $19,4 \%$ empresas con formas societarias.

El presente estudio centró su atención a microempresas limeñas del sector confecciones, cono norte, debido a que estas requieren de la participación de las universidades para remontar el desfase técnico que las agobia, de modo tal que mejoren su competitividad transitando por los caminos de la calidad.

Arroyo (2012), en un diagnóstico situacional de las mypes textiles, revela que $35 \%$ de las PYMEs de Lima cuentan con una capacidad de máquinas mayor a 11, brindando "servicio" a grandes empresas; el $2.24 \%$ de las PYMEs de Lima tienen una capacidad ociosa mayor al $60 \%$, de las cuales el $59 \%$ por falta de carga de trabajo y el $41 \%$ por falta de personal calificado.

* Docente asociado UNMSM. E-mail: otinocog@gmail.com

** Egresado Maestría Logística y Operaciones UNMSM. E-mail: tinocofelix@gmail.com

*** Estudiante del décimo ciclo Ingeniería Textil y Confecciones en la UNMSM.

E-mail: emoscosohuaira@gmail.com 
Por otro lado, $61 \%$ no cuenta con área de corte y 5. $59 \%$ no cuenta con área de acabados.

En el mismo estudio (Arroyo, 2012), se identifican oportunidades de mejora para las mypes textiles: registro de actividades productivas, implementación de las $5 \mathrm{~S}$, elaboración de base de datos de tiempos estándar, mejora continua, determinación de los costos de producción, entre otras.

En resumen, las mipymes en el Perú presentan falencias de organización, gestión de la calidad, hecho que redunda en baja competitividad y menores posibilidades de acceder a mercados más exigentes, como el de las exportaciones.

Ibarra (2010), en su tesis de pregrado, reseña la aplicación de la herramienta de las 5 eses a una empresa de confecciones en México, concluyendo que ello fue de gran importancia, al haberse realizado en cada una de las áreas, contribuyendo a incrementar la calidad del producto final, disminución de pérdidas de tiempo y, sobre todo, el haber conseguido una nueva actitud de los trabajadores.

Juarez (2009) resalta la importancia que tiene esta metodología para mantener un área limpia, reduciendo el riesgo potencial de que se produzcan accidentes; mejorar el bienestar físico y mental del trabajador, se incrementa la vida útil del equipo al evitar su deterioro por contaminación y suciedad; la limpieza conduce a un aumento significativo de la efectividad de equipo.

En tal sentido se formuló como pregunta de investigación:

¿En qué medida la aplicación de la metodología de las $5 \mathrm{~S}$ en una micro empresa de confecciones textiles del Cono Norte de Lima Metropolitana permite mejorar la percepción de una cultura de calidad de sus trabajadores?

\section{METODOLOGÍA}

Se desarrolló una investigación aplicada con diseño preexperimental, tomando como base la totalidad de trabajadores de la microempresa (doce).

El estudio comprendió el diagnóstico del sector, basado tanto en la revisión del estado del arte como en la observación directa. Se aplicaron métodos de la Ingeniería Industrial para la documentación técnica del proceso productivo y del ambiente de trabajo.

Tomando como referencia el trabajo de Gonzales et al., sobre la construcción de un cuestionario para medir el nivel de cultura de calidad en mipymes, se adaptó un instrumento con diez dimensiones y 36 ítems. Ver Tabla 1.

Tabla 1. Dimensiones de las variables en estudio

\begin{tabular}{|c|c|}
\hline Dimensión & Descripción \\
\hline 1 & $\begin{array}{c}\text { Responsabilidad y compromiso de la } \\
\text { gerencia }\end{array}$ \\
\hline 2 & $\begin{array}{c}\text { Responsabilidad y compromiso del } \\
\text { trabajador }\end{array}$ \\
\hline 3 & Confianza en el trabajador \\
\hline 4 & Confianza en la empresa \\
\hline 5 & Satisfacción en el empleo \\
\hline 6 & Comunicación efectiva \\
\hline 7 & Planeación y organización \\
\hline 8 & Visión congruente \\
\hline 9 & Trabajo en equipo \\
\hline 10 & Mejoramiento del servicio \\
\hline
\end{tabular}

Mediante la elaboración de una guía para la aplicación de la metodología de las $5 \mathrm{~S}$ en una mipyme de confecciones se siguió paso a paso dicho proceso, supervisándose el mismo hasta en dos momentos, antes de su concreción final. Ver anexo 1.

La aplicación del instrumento, antes y después de la implementación del método de las $5 \mathrm{~S}$, permitió la recopilación de datos y posterior verificación de hipótesis.

La aplicación de las $5 S$ se relaciona con los conceptos relacionados a cultura de calidad. Según Cantu (2001) cultura de calidad es el conjunto de valores y hábitos que posee una persona, que complementados con el uso de prácticas y herramientas de calidad en el actuar diario, le permiten colaborar con su organización para afrontar los retos que se le presenten en el cumplimiento de su misión.

En la misma dirección, Ghobadian et al. (2004) resaltan que la cultura de calidad se propaga con las prácticas de contratación, los procesos de selección, la inducción y orientación de los nuevos empleados, los programas de desarrollo del personal, la capacitación y el adiestramiento y otras actividades corporativas. 


\section{RESULTADOS}

Como la mayoría de las mimypes de Lima Metropolitana, en la que se desarrolló la investigación los dueños son extrabajadores del sector confecciones que con mucho esfuerzo fueron adquiriendo máquinas y convocando trabajadores para brindar servicios de confección. Posteriormente fueron desarrollando la línea de confección de ropas de baño. Ambas líneas de trabajo coexisten en la actualidad.

El Plano 1 ilustra la distribución de las máquinas en el ambiente de trabajo, típico de una mimype peruana.

La microempresa cuenta con 12 trabajadores que laboran de lunes a sábado. Las labores de gerencia recaen en el dueño y el de administración de la producción en su esposa. Ambos no cuentan con estudios profesionales, basando su accionar en la experiencia.
Además de la mesa de corte, el taller posee 4 máquinas rectas, 3 remalladoras, una recubridora y una collaretera.

\section{IMPLEMENTACIÓN DE LAS 5S}

La principal limitación de la microempresa es el espacio físico, aproximadamente $30 \mathrm{~m}^{2}$, la misma que se hizo extensiva al propósito del estudio. Sobre esa premisa se realizaron diversas reuniones de sensibilización con el personal. Luego les fue entregada la guía de implementación de las $5 \mathrm{~S}$.

\section{INTERPRETACIÓN DE RESULTADOS}

La Tabla 2 resume la variación de la cultura de calidad del conjunto de trabajadores de la microempresa de confecciones, a partir del proceso de implementación de las $5 \mathrm{~S}$ en sus instalaciones.

Plano 1

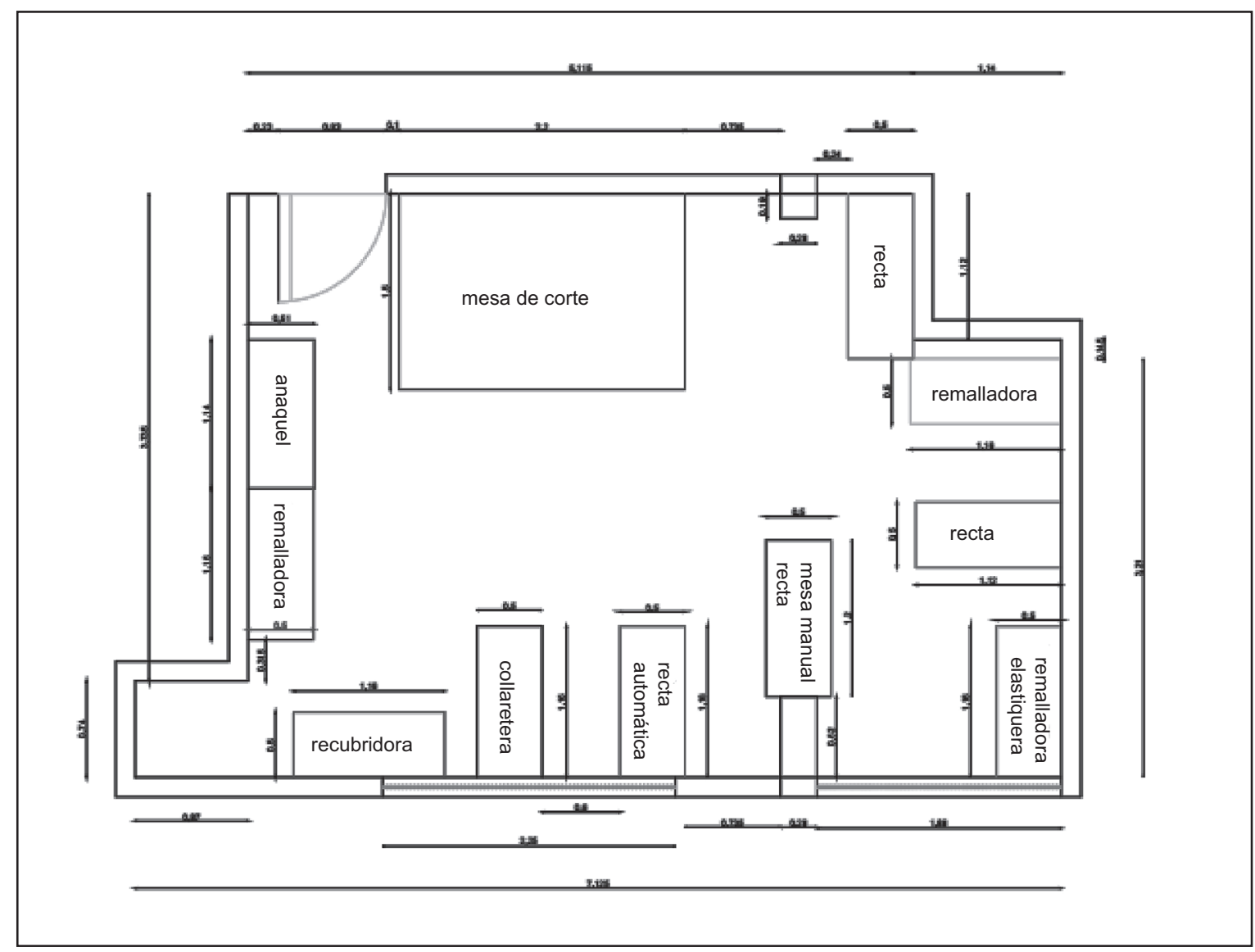


Tabla 2. Estadísticos de muestras relacionadas

\begin{tabular}{|c|c|c|c|c|c|}
\hline \multicolumn{2}{|r|}{ Dimensión } & Media & Desviación & $\mathbf{t}$ & Valor $\mathbf{p}$ \\
\hline \multirow[b]{2}{*}{ D1 } & Responsabilidad y compromiso de la Gerencia antes & 21.50 & 2.067 & \multirow[b]{2}{*}{-13.52} & \multirow[b]{2}{*}{0.000} \\
\hline & $\begin{array}{l}\text { Responsabilidad y compromiso de la Gerencia } \\
\text { después }\end{array}$ & 27.50 & 1.567 & & \\
\hline \multirow{2}{*}{ D2 } & Responsabilidad y compromiso del empleado antes & 14.42 & 1.676 & \multirow{2}{*}{-10.09} & \multirow{2}{*}{0.000} \\
\hline & Responsabilidad y compromiso del empleado después & 18.17 & 1.193 & & \\
\hline \multirow{2}{*}{ D3 } & Confianza en el empleado antes & 11.67 & 1.231 & \multirow{2}{*}{-14.18} & \multirow{2}{*}{0.000} \\
\hline & Confianza en el empleado después & 17.00 & 1.044 & & \\
\hline \multirow{2}{*}{ D4 } & Confianza en la empresa antes & 13.25 & 1.422 & \multirow{2}{*}{-14.12} & \multirow{2}{*}{0.000} \\
\hline & Confianza en la empresa después & 17.67 & 1.371 & & \\
\hline \multirow{2}{*}{ D5 } & Satisfacción en el empleo antes & 7.17 & 1.267 & \multirow{2}{*}{0.90} & \multirow{2}{*}{0.389} \\
\hline & Satisfacción en el empleo después & 6.92 & 1.084 & & \\
\hline \multirow{2}{*}{ D6 } & Comunicación efectiva antes & 12.50 & 1.446 & \multirow{2}{*}{-8.99} & \multirow{2}{*}{0.000} \\
\hline & Comunicación efectiva después & 14.67 & 1.231 & & \\
\hline \multirow{2}{*}{ D7 } & Planeación y organización antes & 9.17 & 1.586 & \multirow{2}{*}{-8.39} & \multirow{2}{*}{0.000} \\
\hline & Planeación y organización después & 13.17 & .718 & & \\
\hline \multirow{2}{*}{ D8 } & Visión congruente antes & 10.42 & .900 & \multirow{2}{*}{-10.00} & \multirow{2}{*}{0.000} \\
\hline & Visión congruente después & 13.75 & .866 & & \\
\hline \multirow{2}{*}{ D9 } & Trabajo en equipo antes & 7.17 & .835 & \multirow{2}{*}{-8.85} & \multirow{2}{*}{0.000} \\
\hline & Trabajo en equipo después & 9.00 & .853 & & \\
\hline \multirow{2}{*}{ D10 } & Mejoramiento continuo antes & 7.92 & .900 & \multirow{2}{*}{-15.13} & \multirow{2}{*}{0.000} \\
\hline & Mejoramiento continuo después & 13.33 & 1.231 & & \\
\hline \multirow{2}{*}{ Total } & Cultura de Calidad Antes & 115.17 & 6.22 & \multirow{2}{*}{-25.36} & \multirow{2}{*}{0.000} \\
\hline & Cultura de Calidad Después & 151.17 & 4.41 & & \\
\hline
\end{tabular}

Fuente: Elaboración propia.

Salvo la dimensión 5, correspondiente a satisfacción con el empleo, la variación es significativa en todas las dimensiones que miden la cultura de calidad en empresas de esta naturaleza. (Valor $p>0.05$ ).

La puntuación total del instrumento revela que la cultura de calidad se eleva de 115.17 a 151.17 puntos y el valor $p$ de 0,000 indica una variación estadísticamente significativa. Se confirma de esta manera la apreciación de Cantu (2006) en el sentido de que la práctica, los valores y el hábito contribuyen al logro de una cultura de calidad.
La mejora notable en aspectos como comunicación, mejora continua y trabajo en equipo del grupo de trabajadores, coincide con lo encontrado por Ibarra (2010) en lo referente a la actitud del recurso humano respecto a la importancia de la calidad.

Los hallazgos de mejora en las dimensiones de responsabilidad y compromiso del empleado y confianza en el empleado coinciden con lo señalado por Juarez (2009) acerca de la importancia de las $5 S$ para mejorar el bienestar físico y mental del trabajador. 


\section{CONCLUSIONES}

La aplicación de la metodología de las $5 \mathrm{~S}$ en una microempresa de confecciones textiles del Cono Norte de Lima Metropolitana permitió mejorar la cultura de calidad en el equipo productivo de una microempresa de confecciones textiles en el Cono Norte de Lima. Al orden y limpieza en el área de trabajo, le sucedió un significativo incremento de una cultura de calidad en dicha entidad.

\section{REFERENCIAS}

[1] Arroyo, Jorge (2012). Diagnóstico situacional de las pymes proveedoras de las grandes empresas de confecciones e identificación de necesidades de capacitación y asistencia técnica. Senati, Perú.

[2] Cantú H. (2005). Desarrollo de una cultura de calidad, México, McGraw Hill.

[3] Cantu, H (2007). Calidad, hábito para la Competitividad. 1.er Congreso Mundial y 7. Encuentro Internacional de Educación Temprana "Ciencia, Conocimiento y educación Temprana". Monterrey, México.

[4] Ghobadian, A. y Gallear, D. (2004). An empirical investigation of the channels that facilitate a total quality culture, Total Quality Management, Octubre 2004, V0l. 15 N. ${ }^{\circ}$ 8, págs. 1047-1067.
[5] Gonzales, Jorge (2010). Construcción del cuestionario para determinar el nivel de Cultura de Calidad en MiPyMEs. Revista Investigación y Ciencias. Número 47, (39-48).

[6] Juarez, H. (2009). Propuesta para implementar metodología $5 \mathrm{~S}$ en el departamento de cobros de la subdelegación Veracruz Norte IMSS. Tesis de Maestría en Gestión de la Calidad Universidad Veracruzana. México.

[7] Produce. Mipyme 2012, estadísticas de la micro, pequeña y mediana empresa. http:// www.produce.gob.pe/remype/data/mype2012. pdf. Consultado en diciembre 2013.

[8] Rios, Germán (2010). Oportunidades y desafíos de financiamiento a las PyMEs. Corporación andina de fomento.. www.caf.com Consultado: Noviembre 2013

[9] SNI (2013) Boletin Sociedad nacional de Industrias: "Sector textil-confecciones aporta con más de S/. 6 mil millones a la economía nacional". Consultado en diciembre 2013. http://www.sni.org.pe/modules. php?name $=$ News\&file $=$ article $\&$ sid $=865$

[10] Stebbing, L. (1997). Aseguramiento de la Calidad. Editorial CECSA, México.

[11] Stuart, Patricia (2011). Pymes responsables. Fondo Editorial Universidad de Lima. Lima. 